\title{
A NICER Look at the AqI X-1 Hard State
}

Bult, Peter; Arzoumanian, Zaven; Cackett, Edward M.; Chakrabarty, Deepto; Gendreau, Keith C.; Guillot, Sebastien; Homan, Jeroen; Jaisawal, Gaurava K.; Keek, Laurens; Kenyon, Steve

Total number of authors:

19

Published in:

The Astrophysical Journal Letters

Link to article, DOI:

10.3847/2041-8213/aac2e2

Publication date:

2018

Document Version

Publisher's PDF, also known as Version of record

Link back to DTU Orbit

Citation (APA):

Bult, P., Arzoumanian, Z., Cackett, E. M., Chakrabarty, D., Gendreau, K. C., Guillot, S., Homan, J., Jaisawal, G. K., Keek, L., Kenyon, S., Lamb, F. K., Ludlam, R., Mahmoodifar, S., Markwardt, C., Miller, J. M., Prigozhin, G., Soong, Y., Strohmayer, T. E., \& Uttley, P. (2018). A NICER Look at the Aql X-1 Hard State. The Astrophysical Journal Letters, 859(1), [L1]. https://doi.org/10.3847/2041-8213/aac2e2

\section{General rights}

Copyright and moral rights for the publications made accessible in the public portal are retained by the authors and/or other copyright owners and it is a condition of accessing publications that users recognise and abide by the legal requirements associated with these rights.

- Users may download and print one copy of any publication from the public portal for the purpose of private study or research.

- You may not further distribute the material or use it for any profit-making activity or commercial gain

- You may freely distribute the URL identifying the publication in the public portal 


\title{
A NICER Look at the Aql X-1 Hard State
}

\author{
Peter Bult $^{1}$, Zaven Arzoumanian ${ }^{1}$, Edward M. Cackett ${ }^{2}$ (1) ${\text {, Deepto } \text { Chakrabarty }^{3} \text { (1), Keith C. Gendreau }}^{1}$,

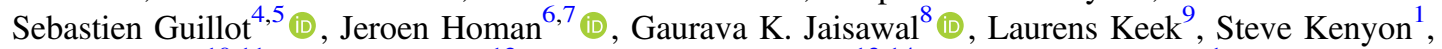 \\ Frederick K. Lamb ${ }^{10,11}$, Renee Ludlam ${ }^{12}$ (D), Simin Mahmoodifar ${ }^{13,14}$ (1) , Craig Markwardt $^{1}$ (D) Jon M. Miller ${ }^{12}$, \\ Gregory Prigozhin $^{3}$, Yang Soong ${ }^{1,9}$, Tod E. Strohmayer ${ }^{13}$, and Phil Uttley ${ }^{15}$ \\ ${ }_{1}^{1}$ Astrophysics Science Division, NASA's Goddard Space Flight Center, Greenbelt, MD 20771, USA \\ ${ }^{2}$ Department of Physics \& Astronomy, Wayne State University, 666 West Hancock, MI 48201, USA \\ ${ }^{3}$ MIT Kavli Institute for Astrophysics and Space Research, Massachusetts Institute of Technology, Cambridge, MA 02139, USA \\ ${ }^{4}$ CNRS, IRAP, 9 avenue du Colonel Roche, BP 44346, F-31028 Toulouse Cedex 4, France \\ ${ }_{6}^{5}$ Université de Toulouse, CNES, UPS-OMP, F-31028 Toulouse, France \\ ${ }^{6}$ Eureka Scientific, Inc., 2452 Delmer Street, Oakland, CA 94602, USA \\ ${ }^{7}$ SRON, Netherlands Institute for Space Research, Sorbonnelaan 2, 3584 CA Utrecht, The Netherlands \\ ${ }^{8}$ National Space Institute, Technical University of Denmark, Elektrovej 327-328, DK-2800 Lyngby, Denmark \\ 9 Department of Astronomy, University of Maryland, College Park, MD 20742, USA \\ ${ }^{10}$ Center for Theoretical Astrophysics and Department of Physics, University of Illinois at Urbana-Champaign, \\ 1110 West Green Street, Urbana, IL 61801-3080, USA \\ ${ }^{11}$ Department of Astronomy,University of Illinois at Urbana-Champaign, 1002 West Green Street, Urbana, IL 61801-3074, USA \\ ${ }^{12}$ Department of Astronomy, University of Michigan, 1085 South University Avenue, Ann Arbor, MI 48109-1107, USA \\ ${ }^{13}$ Astrophysics Science Division and Joint Space-Science Institute, NASA's Goddard Space Flight Center, Greenbelt, MD 20771, USA \\ ${ }^{14}$ CRESST II and the Department of Astronomy, University of Maryland, College Park, MD 20742, USA \\ ${ }^{15}$ Anton Pannekoek Institute, University of Amsterdam, Postbus 94249, 1090 GE Amsterdam, The Netherlands \\ Received 2018 March 26; revised 2018 April 24; accepted 2018 May 6; published 2018 May 17
}

\begin{abstract}
We report on a spectral-timing analysis of the neutron star low-mass X-ray binary (LMXB) Aql X-1 with the Neutron Star Interior Composition Explorer (NICER) on the International Space Station (ISS). Aql X-1 was observed with NICER during a dim outburst in 2017 July, collecting approximately 50 ks of good exposure. The spectral and timing properties of the source correspond to that of a (hard) extreme island state in the atoll classification. We find that the fractional amplitude of the low-frequency $(<0.3 \mathrm{~Hz})$ band-limited noise shows a dramatic turnover as a function of energy: it peaks at $0.5 \mathrm{keV}$ with nearly $25 \% \mathrm{rms}$, drops to $12 \% \mathrm{rms}$ at $2 \mathrm{keV}$, and rises to $15 \% \mathrm{rms}$ at $10 \mathrm{keV}$. Through the analysis of covariance spectra, we demonstrate that band-limited noise exists in both the soft thermal emission and the power-law emission. Additionally, we measure hard time lags, indicating the thermal emission at $0.5 \mathrm{keV}$ leads the power-law emission at $10 \mathrm{keV}$ on a timescale of $\sim 100 \mathrm{~ms}$ at $0.3 \mathrm{~Hz}$ to $\sim 10 \mathrm{~ms}$ at $3 \mathrm{~Hz}$. Our results demonstrate that the thermal emission in the hard state is intrinsically variable, and is driving the modulation of the higher energy power-law. Interpreting the thermal spectrum as disk emission, we find that our results are consistent with the disk propagation model proposed for accretion onto black holes.
\end{abstract}

Key words: accretion, accretion disks - stars: neutron - X-rays: binaries - X-rays: individual (Aql X-1)

\section{Introduction}

The X-ray transient Aquila X-1 is a neutron star low-mass X-ray binary (LMXB) in a $19 \mathrm{hr}$ orbit with a $\sim 1 M_{\odot}$ companion star (Thorstensen et al. 1978; Mata Sánchez et al. 2017). The system shows frequent outbursts with a recurrence rate that evolved from $\sim 125$ days (Priedhorsky \& Terrell 1984) to $\sim 300$ days (Kitamoto et al. 1993), to the approximate $\sim 250$ days in recent times (Campana et al. 2013). These outbursts show a remarkable variation in shape and peak luminosity, with dim outbursts limited to $\leqslant 5 \% L_{\text {edd }}$ and bright outbursts exceeding $30 \% L_{\text {edd }}$, for an Eddington luminosity of $L_{\text {edd }}=3.8 \times 10^{38} \mathrm{erg} \mathrm{s}^{-1}$ (Kuulkers et al. 2003; Campana et al. 2013), and a neutron star mass of 1.4 Solar mass.

Over the course of an outburst Aql X-1 moves through a series of accretion states, each with distinct spectral and timing properties, based on which it has been classified as an "atoll"type neutron star binary (Hasinger \& van der Klis 1989; Reig et al. 2000). At low luminosities the source is in an (extreme) island state, where its energy spectrum is dominated by hard power-law emission and the power spectrum is characterized by broad, large amplitude components. At higher luminosity the source transitions to the so-called "banana" branch, where the energy spectrum pivots to be dominated by its soft thermal components (e.g., Lin et al. 2007). Meanwhile, the variability decreases in amplitude as it shifts to higher frequencies.

Qualitatively similar systematic behavior may be observed in black hole LMXBs (Klein-Wolt \& van der Klis 2008), suggesting that the spectral and timing properties must be a consequence of the accretion process, and be independent of the type of central object. This idea is reinforced by the relation between the characteristic frequencies of the power spectrum, which scale across source types (Wijnands \& van der Klis 1999). Because the variability amplitudes tend to increase as a function of energy (Sobolewska \& Życki 2006), it is thought that the power-law emitting region modulates the observed X-ray flux, although for neutron stars a boundary layer may also play an important role (Gilfanov et al. 2003), and in either case the physical process that sets the frequency may still originate elsewhere in the accretion system.

Advances in spectral-timing analysis of black hole systems has shown that even in the hard state, the soft component may show large amplitude variations at slow timescales (Wilkinson 
\& Uttley 2009), and that this variability leads the modulation of the hard power-law (Uttley et al. 2011). These observations may be explained by an intrinsically variable accretion disk that propagates variability down to the power-law emitting region by modulating the mass accretion rate (Lyubarskii 1997; Kotov et al. 2001; Arévalo \& Uttley 2006).

It is important to confirm whether the spectral-timing characteristics of the band-limited noise are independent of source type, or if they are unique to black hole systems. This type of analysis, however, has been difficult to extend to neutron stars, which are generally fainter in the hard state than their black hole counterparts.

The Neutron Star Interior Composition Explorer (NICER; Gendreau \& Arzoumanian 2017) is an external attached X-ray telescope payload on the International Space Station (ISS). It combines good spectral and timing resolution with excellent sensitivity at $1 \mathrm{keV}$. These properties make NICER a great platform for spectral-timing studies.

In this Letter we report on NICER observations of Aql X-1 during a dim outburst in 2017 July. We present a spectraltiming analysis of all collected data, placing a focus on the behavior of the low-frequency band-limited noise at low energies.

\section{Observations}

NICER's X-ray Timing Instrument (Gendreau et al. 2016) provides an array of 56 co-aligned X-ray concentrator optics, each paired with a silicon drift detector (Prigozhin et al. 2012). Aql X-1 was observed with 52 detectors in operation, as four detectors showed malfunctions prior to launch. Operating in the $0.2-12 \mathrm{keV}$ energy band with a resolution of $\sim 100 \mathrm{eV}$, these detectors provide a combined $\sim 1900 \mathrm{~cm}^{2}$ effective area at $1.5 \mathrm{keV}$. Detected photon events are time-tagged at a relative time resolution of $\sim 40 \mathrm{~ns}$, and have an absolute timing accuracy of $\sim 100 \mathrm{~ns}$ rms.

NICER observed Aql X-1 between 2017 June 20 (MJD 57924) and 2017 July 3 (MJD 57937), collecting $70 \mathrm{ks}$ of unfiltered exposure. In this Letter we consider all of the available data (ObsIDs 0050340101 through 0050340109). We process the data using HEASOFT version 6.22.1 and NICERDAS version 2018-02-22_V002d, selecting only those epochs collected with a pointing offset smaller than 54", more than $40^{\circ}$ away from the bright Earth limb, more than $30^{\circ}$ away from the dark Earth limb, and outside of the South Atlantic Anomaly (SAA). Because the first four ObsIDs were collected while calibration of the X-ray boresight was ongoing, these data have a pointing offset of $70^{\prime \prime}$. As these four ObsIDs contain less than $5 \%$ of the available exposure, we did not attempt to account for the off-axis instrument response, and instead exclude them from the present analysis.

We then construct a light curve using a 12-15 keV energy range. This range lies above the nominal energy band of the instrument because above $12 \mathrm{keV}$ the performance of the optics and detectors has diminished such that essentially no astronomical signal is expected. In the last ObsID, at MJD 57937.545 we observed a $200 \mathrm{~s}$ epoch where the $12-15 \mathrm{keV}$ light curve is significantly different from zero. Correlated with this, the $0.4-10 \mathrm{keV}$ count rate also increases, which we attribute to a high-background interval. This $200 \mathrm{~s}$ epoch is therefore excluded from our analysis. Finally, we use the FTOOL BARYCORR with the radio position of Tudose et al.
(2013) to adjust the photon arrival times to the solar system barycenter (DE405).

After processing we are left with $51 \mathrm{ks}$ of good exposure. Due to the low-Earth orbit of the ISS, our observations are frequently interrupted by Earth occultations and passages through the SAA. Typical continuous exposures are between 100 and $1000 \mathrm{~s}$ in length.

The source-count rate was $\sim 5$ counts/s/detector, yielding a total rate of 270 counts $\mathrm{s}^{-1}$. Because NICER does not provide imaging capabilities, we estimated the background rate from NICER observations of the RXTE background field 5 (Jahoda et al. 2006). We estimate that the background contributed 1.5 counts / $\mathrm{s}$ in the $0.4-10 \mathrm{keV}$ band that we consider in this work.

Two type I X-ray bursts were observed by NICER, the initial analysis of which has been reported by Keek et al. (2018). We excluded the X-ray burst epochs from the analysis presented here, where we defined each epoch as the $200 \mathrm{~s}$ interval beginning $50 \mathrm{~s}$ prior to the burst start time.

\section{Timing}

For the stochastic timing analysis we constructed a light curve at a 1/8192 s time resolution. We divided this light curve into $16 \mathrm{~s}$ segments and, for each segment, computed the power spectrum as well as a soft (1.1-2.0 keV/0.5-1.1 keV) and hard (3.8-6.8 keV/2.0-3.8 keV) color ratio. Because neither the colors nor the light curve count rate showed significant evolution over the course of the observations, we averaged all segments to a single Leahy-normalized power spectrum (Leahy et al. 1983).

Visual inspection of the high-frequency $(\geqslant 2000 \mathrm{~Hz})$ powers confirmed that the power distribution converges to a mean value of 2, consistent with Poisson statistics (Leahy et al. 1983). This is in line with the expectation that deadtime effects are not significant at the recorded count rates due to the modularity of NICER's design. We therefore proceeded by subtracting a constant power level of 2 from our spectrum and renormalized the powers to obtain fractional rms amplitudes with respect to the source rate (van der Klis 1995).

The final power spectrum is modeled using a multiLorentzian component model (Belloni et al. 2002), with each Lorentzian $L\left(\nu ; r, Q, \nu_{\max }\right)$ defined by the characteristic frequency $\nu_{\max }=\nu_{0} \sqrt{1+1 / 4 Q^{2}}$, quality factor $Q$, and centroid frequency $\nu_{0}$. The rms amplitude, $r$, is defined as

$$
r^{2}=\int_{0}^{\infty} L(\nu) d \nu
$$

The power spectrum of Aql X-1 is well described by a fivecomponent model (see Figure 1), the best-fit parameters of which are shown in Table 1. The morphology of the power spectrum is that of the extreme island state (Reig et al. 2004), allowing us to identify the components as (for increasing frequency): the break, low-frequency (LF), hump, low, ${ }^{16}$ and upper $\mathrm{kHz}$ (see van der Klis 2006 for a description of neutron star timing nomenclature). We note that none of the components are sufficiently coherent to qualify as a quasiperiodic oscillation (the threshold is usually set at $Q=2$ ).

We also searched for lower frequency components by considering the averaged power spectrum of $1024 \mathrm{~s}$ segments.

\footnotetext{
16 The low component, traditionally formatted as such, is distinct from the lower $\mathrm{kHz}$ quasi-periodic oscillation (see, e.g., van der Klis 2006).
} 


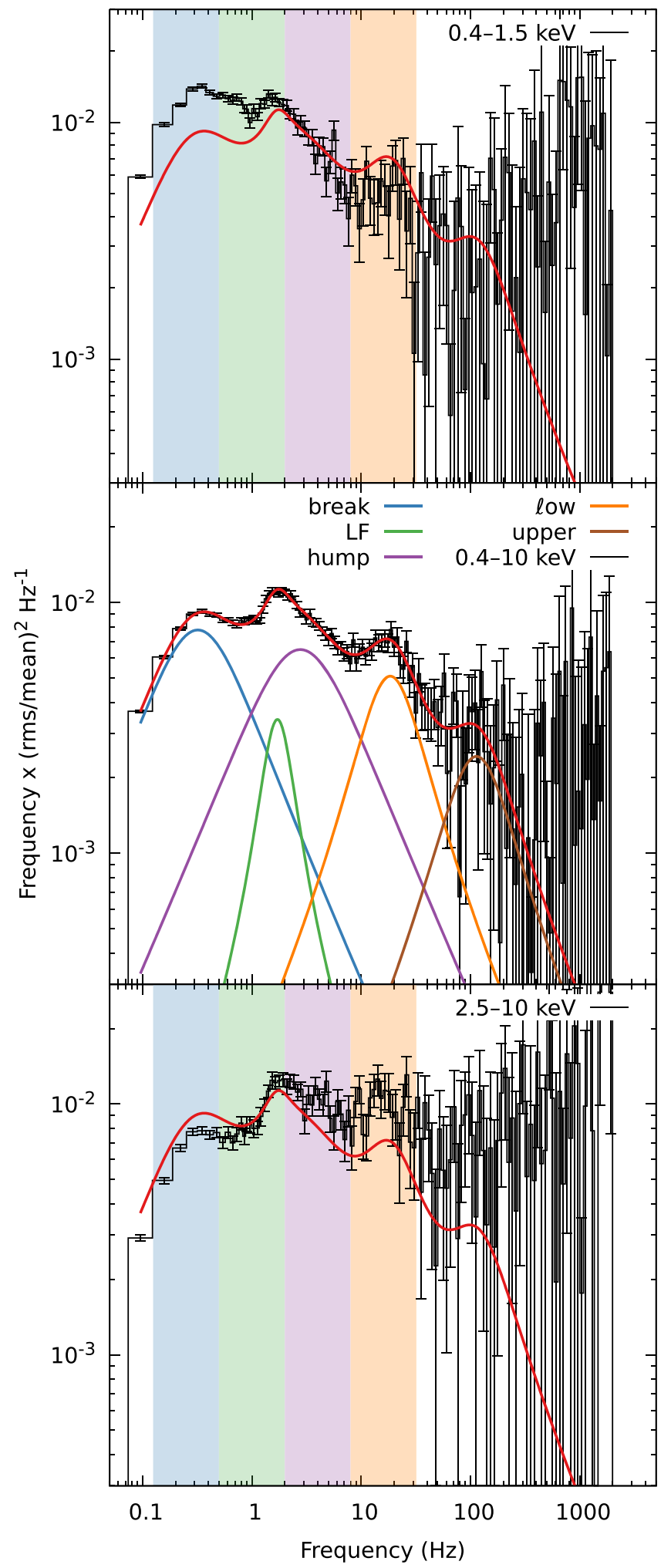

Figure 1. Aql X-1 power spectra and the best-fit $0.4-10 \mathrm{keV}$ model (red), with (top to bottom) the $0.4-1.5 \mathrm{keV}, 0.4-10 \mathrm{keV}$, and $2.5-10 \mathrm{keV}$ band data. Top and bottom panels are included for illustrative purposes only. Shaded regions indicate the covariance frequency intervals (see Section 4).

However, no such slow variability is detected, with the power spectrum remaining roughly constant below the break frequency of $0.3 \mathrm{~Hz}$.

To determine the energy dependence of the power spectrum components we divide the $0.4-10 \mathrm{keV}$ energy band into
Table 1

Power Spectrum Fit Parameters

\begin{tabular}{lrlrl}
\hline \hline & Frequency & $\begin{array}{l}\text { Quality } \\
\text { Factor }\end{array}$ & $\begin{array}{c}\text { Fractional } \\
\text { Amplitude } \\
(\% \mathrm{rms})\end{array}$ & $\chi^{2} /$ dof \\
\hline break & $0.320(10)$ & $0.21(2)$ & $14.2(0.4)$ & \\
LF & $1.71(4)$ & $1.3(0.3)$ & $6.0(1.4)$ & \\
hump & $2.8(0.4)$ & $0.14(8)$ & $13.5(1.0)$ & $115 / 116$ \\
low & $18.4(0.8)$ & $0.57(14)$ & $9.6(0.9)$ & \\
upper & $114(19)$ & $0.5(0.4)$ & $6.9(1.1)$ & \\
\hline
\end{tabular}

Note. Values in parentheses indicate $1 \sigma$ uncertainties.

25 bins, with the energy boundaries chosen such that each bin contains roughly the same number of counts. We then construct an average power spectrum for each bin and fit our multiLorentzian model. As the frequency and quality factor do not change significantly with energy, we keep these parameters fixed, allowing only the rms amplitude of each component to vary.

We find that the amplitude of each component tends to increase approximately linearly with energy above $2 \mathrm{keV}$, consistent with previous findings for Aql X-1 (Cui et al. 1998). Below $2 \mathrm{keV}$, however, we see a sharp rise in the amplitude of the break component, with $15 \% \mathrm{rms}$ at $10 \mathrm{keV}, 12 \% \mathrm{rms}$ at $2 \mathrm{keV}$ and a maximum amplitude of $24.5 \% \mathrm{rms}$ at $0.5 \mathrm{keV}$.

\section{Spectral Timing}

To better characterize the joint spectral and temporal variations, we compute time lags and covariance spectra (Uttley et al. 2014) for four frequency bands between 0.125 and $32 \mathrm{~Hz}$. We scale these frequency ranges geometrically, such that each band is roughly a factor 4 larger than the previous one. These bands correspond approximately with the break, LF, hump, and low components of the power spectrum. A higher frequency band corresponding to the upper $\mathrm{kHz}$ term was left out, as it contained insufficient power to meaningfully constrain the covariance. Spectral-timing products are calculated for narrow energy bands $(\sim 200 \mathrm{eV})$ with respect to a broad $0.5-10 \mathrm{keV}$ reference band by cross-correlating each narrow band with the sum of all other bands (Wilkinson \& Uttley 2009).

The time lags (Figure 2) consistently show that the soft emission leads the hard component. Additionally, we find that the size of the time lag decreases as a function of frequency. The slowest variations show a time difference between 0.5 and $10 \mathrm{keV}$ on the order of $100 \mathrm{~ms}$, whereas the fastest variability arrives nearly simultaneously.

The covariance spectra, expressed as a fraction of the inband count rate, are shown in Figure 3. Consistent with the power spectral amplitudes, we find that the two lowest frequency bands show a dominant peak at $0.5 \mathrm{keV}$ and turnover at $2 \mathrm{keV}$. In the $2-8 \mathrm{~Hz}$ band this soft component is significantly weaker, and in the highest frequency band the turnover at $2 \mathrm{keV}$ is no longer observed. Instead, the two highest frequency bands show a fractional variability that increases approximately linearly from 0.5 to $10 \mathrm{keV}$.

The $0.125-2 \mathrm{~Hz}$ fractional amplitude of the covariance peaks at $17 \% \mathrm{rms}$ at $0.5 \mathrm{keV}$. This is slightly lower than the $25 \%$ peak fractional amplitude measured for the break component at $0.5 \mathrm{keV}$ as derived from the power spectrum. This disparity is 


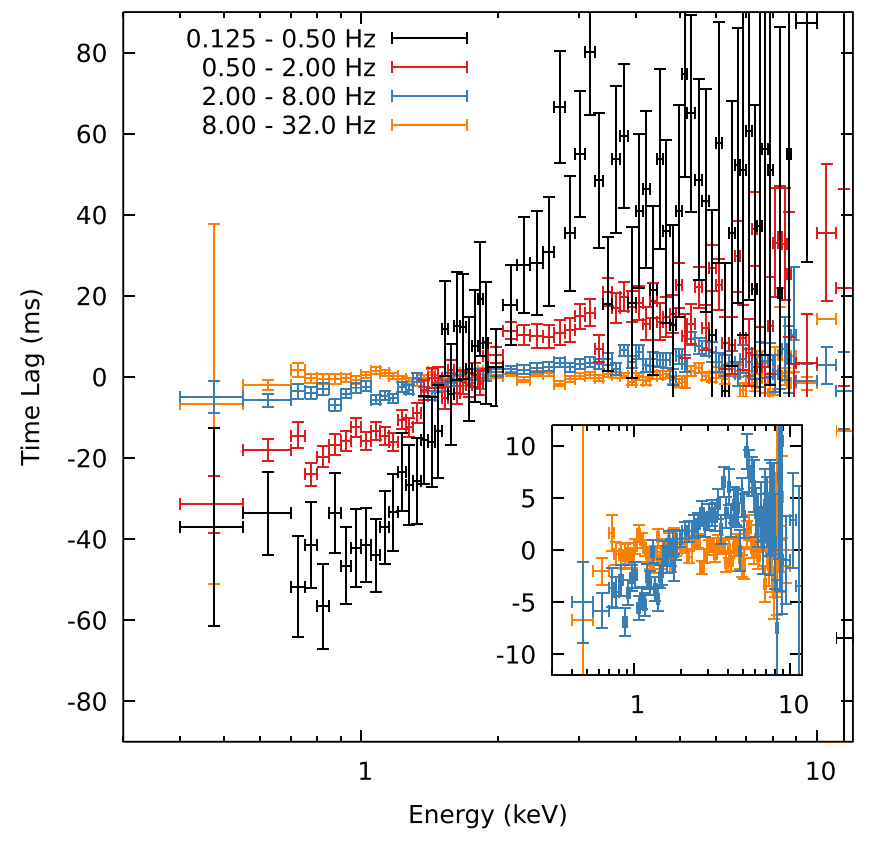

Figure 2. Time lags with respect to a broad reference band in Aql X-1 as a function of energy for four frequency intervals. The inset shows the two highest frequency intervals over a smaller vertical range.

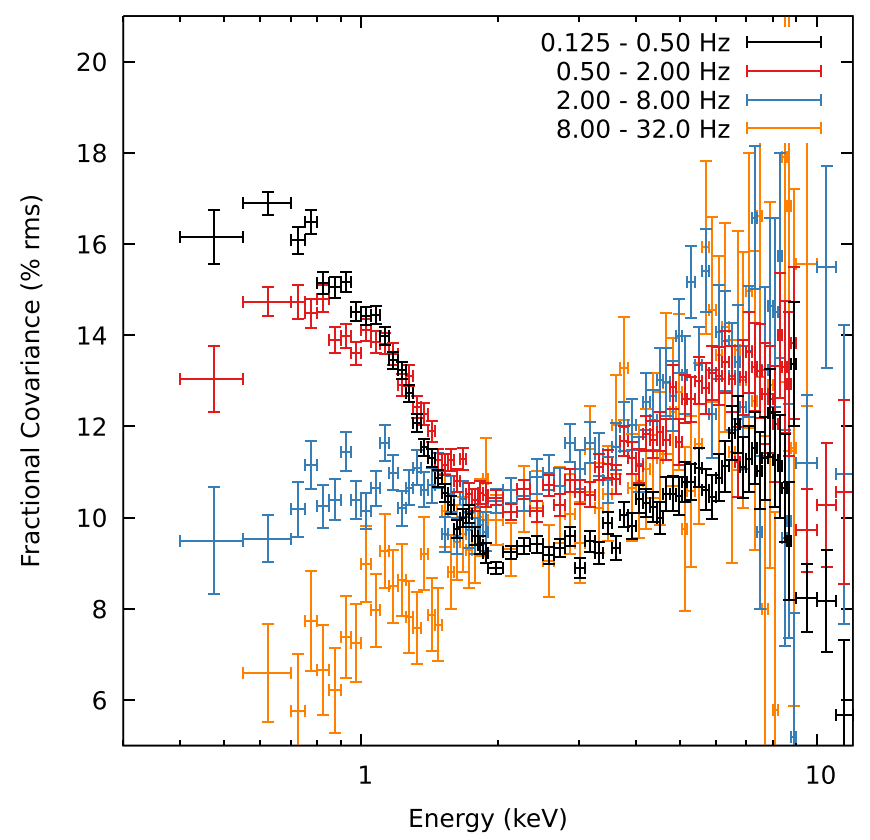

Figure 3. Fractional covariance as a function of energy for four frequency intervals.

due to the different methods used to compute the amplitude. The covariance comes from integrating over a fixed frequency range, whereas the power spectrum amplitude comes from integrating over a Lorentzian profile. The latter captures a broader range of frequencies, and yields a higher integrated power.

In order to determine the association of the variability terms with the spectral components, we normalized the covariance spectra in terms of absolute rms amplitude and folded them with version 0.06 of the NICER instrument response. We then fit the spectra in XSPEC v12.9.1.

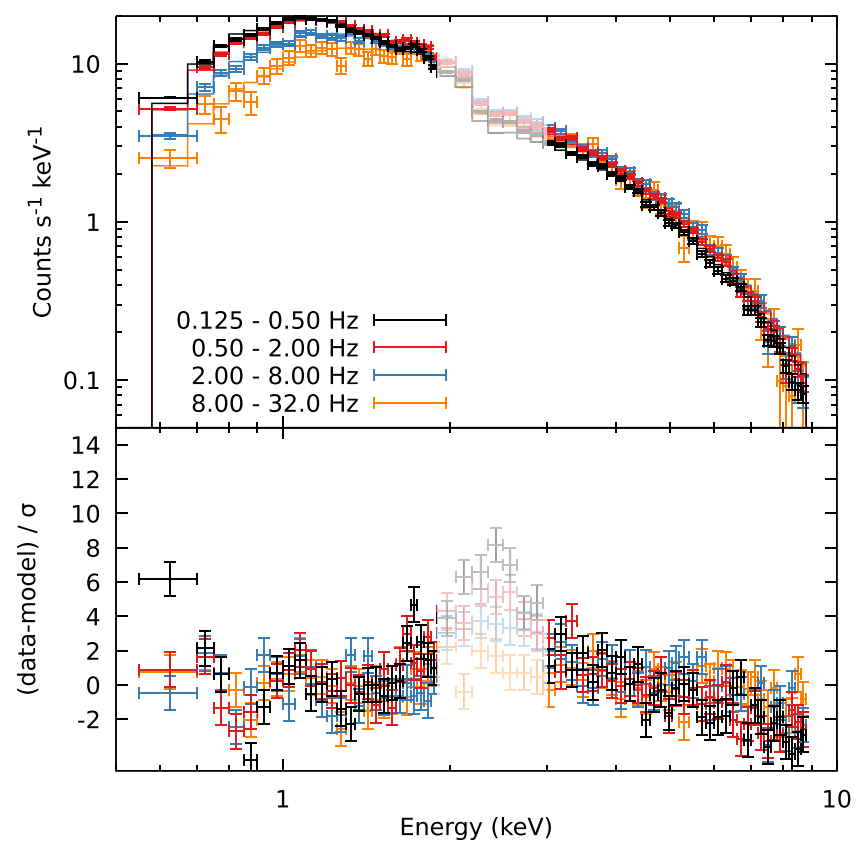

Figure 4. Covariance spectra for four frequency intervals, with (top) the instrument-response folded spectra, and (bottom) the best-fit residuals. Points in the $2-3 \mathrm{keV}$ range were not included in the fit.

We first fit the covariance spectrum of the $8-32 \mathrm{~Hz}$ frequency band, as that has the simplest spectral shape. We model the Galactic absorption using the tbabs model with the abundances of Wilms et al. (2000). At a reduced $\chi^{2}$ of 1.04 (41 degrees of freedom, dof) the spectrum is well described by an absorbed power law model with photon index $\Gamma=1.2 \pm$ 0.1 . The absorption column is $N_{\mathrm{H}}=(6 \pm 1) \times 21 \mathrm{~cm}^{-2}$, which is comparable to values reported from Chandra and XMM-Newton observations (Campana et al. 2014).

Having established a reasonable value for the absorption column, we proceed with performing a joint fit to all four covariance spectra. Compared to the initial $8-32 \mathrm{~Hz}$ best-fit model, each of the other spectra show an unmodeled soft excess below $2 \mathrm{keV}$ and a significant residual near the known instrumental edge at $2.3 \mathrm{keV}$. Because efforts to improve the instrument response matrix are still ongoing, we address the instrumental feature by simply masking the spectral bins in the $2-3 \mathrm{keV}$ range. The soft excess can be adequately described with either a single-temperature blackbody or a multitemperature disk blackbody. Adding an absorbed diskbb term to the spectral model, we obtain a temperature of $k T_{\text {in }}=0.24 \pm 0.01 \mathrm{keV}$ for a reduced $\chi^{2}$ of 1.4 (135 dof; Figure 4). In this fit we kept $N_{\mathrm{H}}$ fixed and tied both disk temperature and the power-law photon index between each of the four covariance spectra. The normalization of the powerlaw approximately the same for each of the four spectra, whereas the normalization of the disk decreases with frequency. The complete set of fit parameters is shown in Table 2.

Finally, we compare our model for the covariance spectra with the energy spectrum of the time-averaged flux. Using all of the considered data we extract an energy spectrum in the 0.4-10 keV range, and extract a spectrum from NICER observations of the RXTE background field 5 using identical filtering criteria. After renormalization, the spectral model used for the covariance roughly matches the shape of the timeaveraged spectrum continuum, although significant residuals 
Table 2

Covariance Spectrum Fit Parameters

\begin{tabular}{|c|c|c|c|c|c|}
\hline Component & Parameter & $0.125-0.5 \mathrm{~Hz}$ & $0.5-2 \mathrm{~Hz}$ & $2-8 \mathrm{~Hz}$ & $8-32 \mathrm{~Hz}$ \\
\hline TBabs & $N_{\mathrm{H}}\left(10^{22} \mathrm{~cm}^{-2}\right)$ & 0.59 & 0.59 & 0.59 & $0.6(1)$ \\
\hline \multirow[t]{2}{*}{ diskbb } & $k T_{\text {in }}(\mathrm{keV})$ & $0.24(1)$ & $0.24(1)$ & $0.24(1)$ & 0.24 \\
\hline & norm $\left(10^{2}\right)$ & $9.6(4)$ & $7.4(3)$ & $3.2(2)$ & $<0.7$ \\
\hline powerlaw & norm $\left(10^{-2}\right)$ & $1.41(5)$ & $1.64(5)$ & $1.67(3)$ & $1.6(1)$ \\
\hline
\end{tabular}

Note. Values in parentheses indicate $90 \%$ confidence intervals, and parameters without a quoted uncertainty were held fixed.

are present. The fit improves slightly by letting the timeaveraged spectrum have a different temperature and photon index, preferring $k T \simeq 0.1 \mathrm{keV}$ and $\Gamma \simeq 1.4$, respectively. Nonetheless, the fit remains only approximate, and could also be described as a disk $(k T \simeq 0.2 \mathrm{keV})$, boundary layer $(k T \simeq 0.8 \mathrm{keV})$, and power-law $(\Gamma \simeq 1.3$ ) akin to the model of Lin et al. (2007). Neither model is statistically acceptable, and, given the early state of the instrument calibration, it is difficult to be more rigorous at this time (see Ludlam et al. 2018 for a discussion of the calibration related uncertainties).

\section{Discussion}

We analyzed the spectral-timing characteristics of Aql X-1 in the hard state and find that the low-frequency band-limited noise exists in both the soft thermal emission and the hard power-law. Additionally, the variability of soft thermal emission leads the correlated modulation of the hard powerlaw. Hence, our results demonstrate that the thermal emission is intrinsically variable and driving the band-limited noise modulation of the power-law.

The spectral-timing characteristics that we observed in Aql X-1 are remarkably similar to those seen in the hard state of the black hole binary GX 339-4 (Uttley et al. 2011), and invite a similar interpretation. In the disk propagation model we can attribute the soft thermal component of the energy spectrum to a cool accretion disk. This disk has intrinsic variations with low frequencies that are observed directly at $0.5 \mathrm{keV}$, where the disk component dominates the spectrum. Through mass-accretion-rate fluctuations, the variability can propagate radially down the accretion flow on a viscous timescale, and modulate a central hot Comptonizing medium (e.g., a corona), which gives rise to increasing variability above $2 \mathrm{keV}$. The time lag between the direct emission from the disk and the reprocessed emission of the modulated Comptonizing region should then scale with distance traveled, which is inversely related to the considered frequency.

We can compare GX 339-4 to Aql X-1 directly by considering the three frequency bands in the $0.125-8 \mathrm{~Hz}$ range, for which covariance and lag measurements exist for both sources. For the black hole the three frequency bands have a 0.5 to $10 \mathrm{keV}$ hard lag of $\sim 150 \mathrm{~ms}, \sim 20 \mathrm{~ms}$, and $\sim 0 \mathrm{~ms}$, respectively. The hard lags measured in Aql X-1 are $\sim 120 \mathrm{~ms}$, $\sim 40 \mathrm{~ms}$, and $\sim 10 \mathrm{~ms}$. Hence, similar frequencies exhibit similar time lags. As a function of frequency the black hole time lags appear to have a steeper slope than the neutron star lags, albeit tentatively so.

The fractional covariances are less straightforward to compare, as spectral-timing studies of black holes do not typically consider fractional amplitudes. However, we can express the soft excess of our $0.125-0.5 \mathrm{~Hz}$ covariance spectrum as a ratio, by dividing the spectrum with the powerlaw model component only. We then find that the soft excess of Aql X-1 has a ratio value of approximately 2.5 at $0.5 \mathrm{keV}$. This is again similar to the GX 339-4 measurements of Uttley et al. (2011).

In contrast to GX 339-4, we find that the photon index of the covariance spectrum in Aql X-1 is comparatively low. However, Aql X-1 has shown a brief epoch of coherent pulsations (Casella et al. 2008) and spectral studies of its soft state suggest a truncated accretion disk (King et al. 2016; Ludlam et al. 2017). This suggests that the accretion flow in Aql X-1 may be interacting with a dynamically relevant magnetosphere, which could increase the electron temperature associated with the Comptonizing region, and possibly cause the flatter power-law. We warn, however, that the photon index that we report should be considered with some caution. A power-law model to describe the spectrum is almost certainly too simplistic, and is used only because our covariance spectra could not distinguish between more physically motivated models. A more sensitive approach would be to obtain the absorption column $N_{\mathrm{H}}$ and photon index from the timeaveraged flux. Given the early state of the instrument calibration, however, such a detailed study of the timeaveraged flux cannot be robustly performed at this time.

It is somewhat surprising that in the presence of a neutron star, the hard state spectral-timing properties of Aql X-1 are so similar to that of GX 339-4. This may suggest that the emission associated with the neutron star is not well correlated with the band-limited noise, or that the spectral-timing signature of that emission is very similar to that of the disk propagation model. We note, for instance, that detailed modeling of a boundary layer surrounded by a Comptonizing medium has been done in the context of Z-sources. Such work demonstrated that if the electron scattering optical depth of the Comptonizing medium is modulated periodically, the emergent $\mathrm{X}$-ray spectrum has similar characteristics to what we observe: time lags between the emission above and below a pivot energy $\left(E_{P}\right)$, and a minimum in the fractional variability at $E_{P}$ (Miller \& Lamb 1992; Lee \& Miller 1998). We venture that a stochastic modulation of the optical depth may produce similar spectral-timing characteristics as seen in those calculations. The pivot energy would then be set by the shape of the Comptonized spectrum, and the power spectrum of the stochastic modulation. The slow modulation would still be driven by mass-accretion-rate fluctuation in the disk. Higher frequency fluctuations would then originate predominantly within the corona, and hence show increasing fractional covariance at high energies without generating significant time lags. This picture does not fully address the similarities with black hole systems, but is at least qualitatively consistent with 
the spectral-timing properties that we observe in Aql X-1. We therefore suggest that it may be worthwhile to explore whether such a model could reproduce the pivot energy and contribute to the spectral-timing properties that we measured.

In summary, we have established that band-limited noise in the hard state of Aql X-1 is driven by the soft thermal component. The striking similarities of our results with the black hole GX 339-4 suggest that this soft component is most likely the cool accretion disk, and that the spectral-timing features are due to propagating mass-accretion-rate fluctuations. However, we note that the complex interactions of the accretion flow with the neutron star surface and magnetosphere will require more detailed modeling to explain the similarity of observed spectral-timing features. We suggest that further spectral-timing studies of neutron stars, enabled by NICER, will provide fertile ground for these efforts.

This work was supported by NASA through the NICER mission and the Astrophysics Explorers Program, and made use of data and software provided by the High Energy Astrophysics Science Archive Research Center (HEASARC). P.B. was supported by an NPP fellowship at NASA Goddard Space Flight Center. E.M.C. gratefully acknowledges support from the National Science Foundation through CAREER award number AST-1351222.

Facilities: ADS, HEASARC, NICER.

\section{ORCID iDs}

Edward M. Cackett (ํ) https://orcid.org/0000-0002-8294-9281 Deepto Chakrabarty (1) https://orcid.org/0000-00018804-8946

Sebastien Guillot (1) https://orcid.org/0000-0002-6449-106X

Jeroen Homan (1) https://orcid.org/0000-0001-8371-2713

Gaurava K. Jaisawal (1) https://orcid.org/0000-0002-

6789-2723

Renee Ludlam (i) https://orcid.org/0000-0002-8961-939X

Simin Mahmoodifar (10) https://orcid.org/0000-0003-

2386-1359

Craig Markwardt (1) https://orcid.org/0000-0001-9803-3879

\section{References}

Arévalo, P., \& Uttley, P. 2006, MNRAS, 367, 801

Belloni, T., Psaltis, D., \& van der Klis, M. 2002, ApJ, 572, 392

Campana, S., Brivio, F., Degenaar, N., et al. 2014, MNRAS, 441, 1984

Campana, S., Coti Zelati, F., \& D’Avanzo, P. 2013, MNRAS, 432, 1695

Casella, P., Altamirano, D., Patruno, A., Wijnands, R., \& van der Klis, M. 2008, ApJL, 674, L41

Cui, W., Barret, D., Zhang, S. N., et al. 1998, ApJL, 502, L49

Gendreau, K., \& Arzoumanian, Z. 2017, NatAs, 1, 895

Gendreau, K. C., Arzoumanian, Z., Adkins, P. W., et al. 2016, Proc. SPIE, 9905, $99051 \mathrm{H}$

Gilfanov, M., Revnivtsev, M., \& Molkov, S. 2003, A\&A, 410, 217

Hasinger, G., \& van der Klis, M. 1989, A\&A, 225, 79

Jahoda, K., Markwardt, C. B., Radeva, Y., et al. 2006, ApJS, 163, 401

Keek, L., Arzoumanian, Z., Bult, P., et al. 2018, ApJL, 855, L4

King, A. L., Tomsick, J. A., Miller, J. M., et al. 2016, ApJL, 819, L29

Kitamoto, S., Tsunemi, H., Miyamoto, S., \& Roussel-Dupre, D. 1993, ApJ, 403, 315

Klein-Wolt, M., \& van der Klis, M. 2008, ApJ, 675, 1407

Kotov, O., Churazov, E., \& Gilfanov, M. 2001, MNRAS, 327, 799

Kuulkers, E., den Hartog, P. R., in't Zand, J. J. M., et al. 2003, A\&A, 399, 663

Leahy, D. A., Darbro, W., Elsner, R. F., et al. 1983, ApJ, 266, 160

Lee, H. C., \& Miller, G. S. 1998, MNRAS, 299, 479

Lin, D., Remillard, R. A., \& Homan, J. 2007, ApJ, 667, 1073

Ludlam, R. M., Miller, J. M., Arzoumanian, Z., et al. 2018, ApJL, 858, L5

Ludlam, R. M., Miller, J. M., Degenaar, N., et al. 2017, ApJ, 847, 135

Lyubarskii, Y. E. 1997, MNRAS, 292, 679

Mata Sánchez, D., Muñoz-Darias, T., Casares, J., \& Jiménez-Ibarra, F. 2017, MNRAS, 464, L41

Miller, G. S., \& Lamb, F. K. 1992, ApJ, 388, 541

Priedhorsky, W. C., \& Terrell, J. 1984, ApJ, 280, 661

Prigozhin, G., Gendreau, K., Foster, R., et al. 2012, Proc. SPIE, 8453, 845318

Reig, P., Méndez, M., van der Klis, M., \& Ford, E. C. 2000, ApJ, 530, 916

Reig, P., van Straaten, S., \& van der Klis, M. 2004, ApJ, 602, 918

Sobolewska, M. A., \& Życki, P. T. 2006, MNRAS, 370, 405

Thorstensen, J., Charles, P., \& Bowyer, S. 1978, ApJL, 220, L131

Tudose, V., Paragi, Z., Yang, J., et al. 2013, ATel, 5158, 1

Uttley, P., Cackett, E. M., Fabian, A. C., Kara, E., \& Wilkins, D. R. 2014, A\&ARv, 22, 72

Uttley, P., Wilkinson, T., Cassatella, P., et al. 2011, MNRAS, 414, L60

van der Klis, M. 1995, in The Lives of the Neutron Stars, Vol. 450, ed. M. A. Alpar, U. Kiziloglu, \& J. van Paradijs (Dordrecht: Kluwer), 301 van der Klis, M. 2006, in Compact Stellar X-ray Sources, ed.

W. H. G. Lewin \& M. van der Klis (Cambridge: Cambridge Univ. Press), 39 Wijnands, R., \& van der Klis, M. 1999, ApJ, 514, 939

Wilkinson, T., \& Uttley, P. 2009, MNRAS, 397, 666

Wilms, J., Allen, A., \& McCray, R. 2000, ApJ, 542, 914 